\title{
Compensatory patterns of collateral flow in stroke patients with unilateral and bilateral carotid stenosis
}

\author{
Hui Fang ${ }^{1,2}$, Bo Song ${ }^{1}$, Bo Cheng ${ }^{2}$, Ka Sing Wong ${ }^{2}$, Yu Ming Xu' , Stella Sin Yee Ho ${ }^{3^{*}}$ and Xiang Yan Chen ${ }^{2^{*}}$
}

\begin{abstract}
Background: Collateral pathways are important in maintaining adequate cerebral blood flow in patients with carotid stenosis. We aimed to evaluate the hemodynamic patterns in relation to carotid stenosis in acute stroke patients.

Methods: Consecutive 586 stroke patients in a hospital based cohort were included in the present study. Carotid duplex was performed to identify patients with absolute minimal diameter reductions of $50 \%$ or greater in their internal carotid arteries (ICAs). Color velocity imaging quantification ultrasound (CVIQ) was used to measure extracranial arterial blood flow volume (BFV) in bilateral common carotid arteries (CCAs) and bilateral vertebral arteries (VAs). The absolute values of BFV and the ratios were compared between patients with and without ICA stenosis.

Results: Among 586 acute ischemic stroke patients (mean age: $67.5 \pm 12.4 \mathrm{y}$ ), ICA stenosis was detected in 112 patients (19.1\%), including unilateral ICA stenosis in 81 patients (13.8\%) and bilateral ICA stenosis in 31 patients (5.3\%). Among patients with unilateral ICA stenosis, the BFV in contralateral CCA was significantly higher than that in ipsilateral CCA ( $325.5 \pm 99.8 \mathrm{~mL} / \mathrm{min}$ vs. $242.2 \pm 112.2 \mathrm{~mL} / \mathrm{min}, P<0.001$ ). Among patients with bilateral ICA stenosis, the sum of BFV in bilateral VAs accounted for $22 \%$ of the whole cerebral blood flow, which was significantly higher than that in those without ICA stenosis $(14.8 \%, P<0.001)$ or with unilateral ICA stenosis $(16.9 \%, P=0.007)$.
\end{abstract}

Conclusions: In patients with unilateral carotid stenosis, contralateral carotid blood flow increases to compensate decreased blood flow, while posterior circulation may compensate for the decreased brain perfusion in those with bilateral carotid stenosis.

Keywords: Collateral circulation, Carotid stenosis, Stroke, Ultrasonography

\section{Background}

According to the results from large randomized trials, carotid endarterectomy has become the accepted standard treatment of choice for patients with recently symptomatic carotid stenosis, and selected patients can benefit from revascularization with endarterectomy or stenting [1]. However, limited information has been obtained regarding optimal selection of revascularization candidates. The current approach to patient selection is primarily based on

\footnotetext{
* Correspondence: stellaho@cuhk.edu.hk; fiona.xy2000@gmail.com ${ }^{3}$ Department of Imaging and Interventional Radiology, Faculty of Medicine, Prince of Wales Hospital, The Chinese University of Hong Kong, Shatin, New Territories, Hong Kong

${ }^{2}$ Department of Medicine and Therapeutics, Faculty of Medicine, Prince of Wales Hospital, The Chinese University of Hong Kong, Shatin, New Territories, Hong Kong

Full list of author information is available at the end of the article
}

anatomical identification of luminal stenosis, without taking into account compensatory mechanisms, poststenotic flow, downstream perfusion status, and presence of collateral circulation [2-4], which thus may be insufficient and misleading. It is well recognized that simply an anatomically luminal stenosis might not always lead to impaired cerebral hemodynamics and hypoperfusion in the distal cerebral circulation $[5,6]$. Therefore, it is necessary to develop imaging techniques that can be used to evaluate the downstream hemodynamics and cerebral perfusion of extracranial steno-occlusive disease [7].

Color velocity imaging quantification (CVIQ) ultrasound has been proved to be a noninvasive technique that can 
reveal extracranial arterial blood flow volume, which is an indicator of cerebral perfusion [8-12]. Our previous studies demonstrated that poststroke extracranial arterial blood flow volume estimated by CVIQ is an independent predictor of functional outcome and recurrent stroke $[11,12]$. In addition, it is important to assess the contribution of the contralateral ICA, the ipsilateral ECA, and the vertebral arteries to the collateral pathways in patients with symptomatic ICA stenosis [13-15]. The quantitative measurement of carotid and vertebral blood flow volume (BFV), which has been suggested as an estimate of total cerebral BFV is clinically of great value in evaluating the presence of collateral pathways and hemodynamic conditions in patients with carotid stenosis [16-18]. Furthermore, compared with other quantitative examinations to determine cerebral perfusion as MRI perfusion, CT perfusion, Single Photon Emission Computed Tomography (SPECT), CVIQ is a noninvasive, easy to access, economic, and free of any known harm techniques which requires no contrast agent. In the present study, we evaluated the hemodynamic patterns in acute stroke patients with unilateral and bilateral internal carotid artery (ICA) stenosis by using CVIQ ultrasound.

\section{Methods}

\section{Patients}

It was a hospital based cohort study including consecutive stroke patients admitted to the Prince of Wales Hospital (PWH) from January 1, 1998 to December 31, 2000. The study was approved by the Joint Chinese University of Hong Kong - New Territories East Cluster Clinical Research Ethics Committee (The Joint CUHKNTEC CREC). Informed written consent was obtained from each patient before blood flow volume measurement. The patients were recruited according to the inclusion criteria: (1) Sudden onset of neurological symptoms that persisted for $\geq 24 \mathrm{~h}$ based on the World Health Organization criteria; (2) Diagnosis confirmed by a CT scan of the head that excluded hemorrhage or other alternative diagnosis. Patients with cardioembolic stroke according to the Trial of ORG 10172 in Acute Stroke Treatment (TOAST) criteria and those who were clinically unstable for transfer to the ultrasound vascular laboratory for the study were excluded.

\section{Data acquisition}

The assessment of carotid stenosis and measurement of BFV was performed as described previously $[8,9,11,12]$. A 7.5-MHz linear transducer of a Philips SD800 ultrasound unit (Best, the Netherlands) which has a standard feature of duplex Doppler imaging as well as a color velocity imaging quantification (CVIQ) software for blood flow volume measurement was used. Carotid duplex was performed to determine the presence of ICA stenosis $(\geq 50 \%)$. Carotid stenosis was defined as the ratio of the peak systolic velocity at the internal carotid artery to that at the ipsilateral common carotid artery greater than 1.5 or direct measurement of the stenosis greater than $50 \%$ on the static image if the true and residual lumen could be clearly depicted. The ipsilateral-tocontralateral CCA blood flow volume ratio was defined as the ratio of the BFV in the ipsilateral CCA to that in the contralateral CCA.

CVIQ technique was performed to measure BFV in bilateral common carotid arteries (CCAs) and bilateral vertebral arteries (VAs). The average of 3 repeated measurements was taken as the BFV of an individual artery. The intra- and interobserver reproducibility was tested by two operators utilizing the standardized technique. The intraclass correlation coefficient for interobserver reproducibility between operators A and B was 0.75 and those for intraobserver reproducibility of operators A and B were 0.88 and $0.93(p<0.001)$, respectively.

According to the presence of $\geq 50 \%$ carotid stenosis, patients were divided into three groups: patients without carotid stenosis as control group, those with unilateral carotid stenosis and those with bilateral carotid stenosis.

\section{Statistical analysis}

All values were indicated as mean \pm standard deviation, and tested for normal distribution by the KolmogorovSmirnov procedure. Two-tailed Student $t$ test was performed to compare the values with normal distribution. $P$ value of less than 0.05 was considered to be significant. Spearman correlation was used to assess the relationship between degree of artery stenosis and blood flow volume. The absolute values and the ratios of BFV in different arteries were compared between the patients with unilateral and bilateral ICA stenosis. All analyses were performed with SPSS.

\section{Results \\ Baseline characteristics}

During the study period, 586 acute ischemic stroke patients were recruited (mean age, $67.5 \pm 12.4 y$ ). Unilateral ICA stenosis was detected in $81(13.8 \%)$ patients and bilateral ICA stenosis in 31 (5.3\%) patients by using carotid duplex with 1 week after stroke onset. The baseline characteristics of stroke patients were summarized in Table 1. Compared with patients without carotid stenosis, patients with unilateral or bilateral carotid stenosis were older $(P=0.002)$, and had a higher rate of male $(P=0.008)$, hypertension $(P=0.002)$ and previous cerebral vascular diseases $(p=$ 0.035 ). However, stroke patients with unilateral and bilateral carotid stenosis had comparable prevalence of age and risk factors (Table 2). 
Table 1 Baseline Characteristics of the whole stroke patients and comparisons between patients with and without ICA stenosis

\begin{tabular}{lllll}
\hline Baseline characteristics & Total $N=586$ & Patients without ICA stenosis $n=474$ & Patients with ICA stenosis $n=112$ & $P$ value \\
\hline Male sex, \% & $316,53.9 \%$ & $243,51.3 \%$ & $73,65.2 \%$ & 0.008 \\
Mean age, y & $67.5 \pm 12.4$ & $66.7 \pm 12.8$ & $70.7 \pm 9.7$ & 0.002 \\
Hypertension, \% & $309,52.7 \%$ & $235,49.6 \%$ & $74,66.1 \%$ & 0.002 \\
Diabetes Mellitus, \% & $181,30.9 \%$ & $139,29.3 \%$ & $42,37.5 \%$ & 0.092 \\
Hyperlipidemia, \% & $192,32.8 \%$ & $154,32.5 \%$ & $38,33.9 \%$ & 0.783 \\
Smoking, \% & $162,27.6 \%$ & $125,26.4 \%$ & $37,33 \%$ & 0.156 \\
Ischemic Heart Disease, \% & $227,38.7 \%$ & $175,36.9 \%$ & $52,46.4 \%$ & $37,33 \%$ \\
Previous Stroke or TIA, \% & $148,25.3 \%$ & $111,23.4 \%$ & $680 \pm 175.7$ & 0.063 \\
Blood Flow Volume & $727.2 \pm 188.3$ & $738.3 \pm 189.6$ & $559.1 \pm 156.9$ & 0.035 \\
Bilateral CCAs & $615.8 \pm 167.6$ & $629.2 \pm 167.4$ & $123.2 \pm 67.3$ & 0.003 \\
Bilateral VAs & $111.8 \pm 55.8$ & $109.1 \pm 52.5$ & $82.2 \pm 9.3$ & 0.001 \\
Bilateral CCA\% & $84.6 \pm 6.8$ & $85.2 \pm 5.9$ & $18.3 \pm 8.6$ & 0.016 \\
Bilateral VA\% & $15.5 \pm 6.6$ & $14.8 \pm 5.9$ & $<.001$ \\
\hline
\end{tabular}

\section{Hemodynamic data}

Comparisons of hemodynamic data between stroke patients without ICA stenosis and with unilateral or bilateral ICA stenosis were described in Table 3. Compared with those without carotid stenosis $(738.3 \pm 189.6 \mathrm{ml} / \mathrm{min})$, the total BFV decreased in patients with unilateral carotid stenosis $(681.7 \pm 175.5 \mathrm{ml} / \mathrm{min} ; p=0.012)$ and tended to decrease in those with bilateral carotid stenosis $(675.6 \pm 179.1 \mathrm{ml} / \mathrm{min}$; $p=0.074)$. The sum of BFV in bilateral VAs increased but did not reach statistical significance compared with patients without ICA stenosis $(114.3 \pm 64.4 \mathrm{ml} / \mathrm{min}$ vs. $109.1 \pm$ $52.5 \mathrm{ml} / \mathrm{min}, P=0.421$ ). However, the percentage of BFV in bilateral VAs accounted for almost $16.9 \%$ of the whole brain BFV, which was significantly higher than $14.8 \%$ in those without ICA stenosis $(P=0.036)$.
Among stroke patients with unilateral ICA stenosis, the absolute values of BFV in ipsilateral CCA or the ratios decreased with the increasing severity of ICA stenosis, as shown in Figs. 1 and 2. The absolute values of BFV in contralateral CCA, as well as the ratios of contralateral CCA BFV, was significantly higher than that in ipsilateral CCA $(325.5 \pm 99.8 \mathrm{~mL} / \mathrm{min}$ vs. $242.2 \pm 112.2 \mathrm{~mL} / \mathrm{min}, P<$ $0.001 ; 48.5 \pm 12.2 \%$ vs. $34.7 \pm 12.5 \%, P<0.001$ ) (Table 4 ). However, there were 25 (30.9\%) patients with significant increase in BFV in ipsilateral CCA $(323.8 \pm 75.4)$, implying collateral pathways from inpsilateral ECA artery.

Compared to stroke patients without ICA stenosis, the absolute values of bilateral CCA BFV in patients with bilateral ICA stenosis and the ratios decreased significantly $(567.3 \pm 160.3$ vs. $629.2 \pm 167.4, P=0.002 ; 83.0 \pm 8.0 \%$

Table 2 Comparisons of clinical features between stroke patients with unilateral and bilateral ICA stenosis

\begin{tabular}{llll}
\hline & Patients with unilateral ICA stenosis $n=81$ & Patients with bilateral ICA stenosis $n=31$ & $P$-value \\
\hline Male sex, \% & $53,65.4 \%$ & $20,64.5 \%$ & $71.2 \pm 9.8$ \\
Mean age, y & $70.6 \pm 9.7$ & $18,58.1 \%$ & 0.927 \\
Hypertension, \% & $56,69.1 \%$ & $11,35.5 \%$ & 0.268 \\
Diabetes Mellitus, \% & $31,38.3 \%$ & $8,25.8 \%$ & 0.785 \\
Hyperlipidemia, \% & $30,37 \%$ & $10,32.3 \%$ & $13,41.9 \%$ \\
Smoking, \% & $27,33.3 \%$ & $9,29 \%$ & 0.379 \\
Ischemic Heart Disease, \% & $39,48.1 \%$ & $22,71 \%$ & 0.914 \\
Previous Stroke or TIA, \% & $28,34.6 \%$ & $675.6 \pm 179.1$ & 0.555 \\
Patients with carotid stenosis $>70 \%, \%$ & $43,53.1 \%$ & $146.2 \pm 70.4$ & 0.577 \\
Blood Flow Volume & $681.7 \pm 175.5$ & $22 \pm 8.9$ & 0.065 \\
BA & $114.3 \pm 64.4$ & $537.4 \pm 147.9$ & 0.872 \\
BA\% & $16.8 \pm 8.1$ & $79.6 \pm 11.8$ & 0.024 \\
Bilateral CCAs & $567.3 \pm 160.3$ & 0.004 \\
Bilateral CCAs\% & $83.2 \pm 8.1$ & 0.369 \\
\hline
\end{tabular}


Table 3 Comparisons of hemodynamic data between stroke patients without ICA stenosis and those with unilateral or bilateral ICA stenosis

\begin{tabular}{lccccc}
\hline & $\begin{array}{c}\text { Patients without ICA stenosis } \\
n=474\end{array}$ & $\begin{array}{c}\text { Patient with unilateral ICA stenosis } \\
n=81\end{array}$ & $\begin{array}{c}P \text { value* } \\
\text { Total BFV }\end{array}$ & $\begin{array}{l}\text { Patient with bilateral ICA stenosis } \\
n=31\end{array}$ & $P$ value*** \\
Bilateral CCA & $738.3 \pm 189.6$ & $681.7 \pm 175.5$ & 0.012 & $675.6 \pm 179.1$ & 0.074 \\
Bilateral CCA\% & $629.2 \pm 167.4$ & $567.3 \pm 160.3$ & 0.002 & $537.4 \pm 147.9$ & 0.003 \\
BFV in bilateral VAs & $85.0 \pm 6.0$ & $83.0 \pm 8.0$ & 0.036 & $79.5 \pm 11.8$ & 0.013 \\
\% BFV in bilateral VAs & $109.1 \pm 52.5$ & $114.3 \pm 64.4$ & 0.421 & $146.2 \pm 70.4$ & $<0.001$ \\
\hline
\end{tabular}

*indicates the comparison between patients with unilateral ICA stenosis and patients without ICA stenosis

**indicates the comparison between patients with bilateral ICA stenosis and patients without ICA stenosis

vs. $85.0 \pm 6.0 ; P=0.036$ ). The sum of BFV in bilateral VAs increased significantly compared with patients without ICA stenosis $(146.2 \pm 70.4 \mathrm{ml} / \mathrm{min}$ vs. $109.1 \pm$ $52.5 \mathrm{ml} / \mathrm{min}, P<0.001$ ) and accounted for almost $22 \%$ of the whole brain BFV, which was significantly higher than $14.8 \%$ in those without ICA stenosis $(P<0.001)$.

\section{Discussion}

In patients with unilateral carotid artery stenosis, evidence is accumulating to suggest that compromised hemodynamic brain state is associated with an increased risk of stroke than if they have a normal brain perfusion [19-21]. In recent years, the controversy as to whether hypoperfusion as a major cause of stroke is attributable to lacking of good standard of hemodynamic stroke and the interaction of hypoperfusion and embolism to cause ischemic stroke in many patients $[14,22]$. In this study, we found that patients with ICA stenosis suffered from compromised global perfusion, compared with patients without ICA stenosis and the diminished perfusion was associated with the severity of ICA stenosis. In addition, our results found that stroke patients with unilateral stenosis had significantly more flow in the contralateral CCA than in the ipsilateral CCA, which reflects the existence of collateral blood flow to the supply territory of the ipsilateral CCA to maintain cerebral blood flow. Meanwhile, 25 patients had significant increase in BFV in ipsilateral CCA, implying that there were also pathways from inpsilateral ECA artery together with contralateral CCA to form the collateral circulation of stroke patients with carotid stenosis.

The most important finding of the present study was that CVIQ was able to assess the compensatory patterns of collateral flow from the contralateral ICA and bilateral

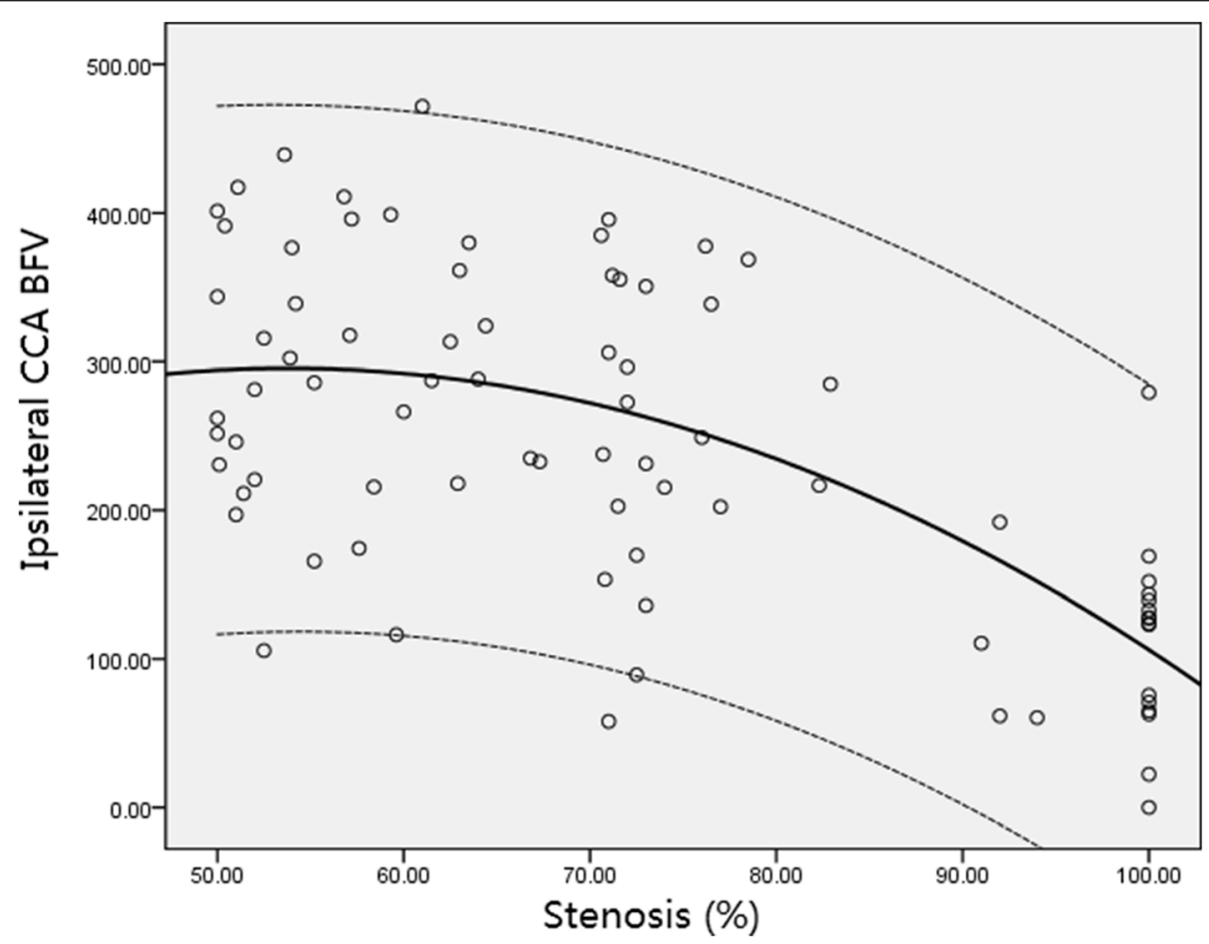

Fig. 1 A decrease in ipsilateral CCA blood flow is significantly associated with increasing percentage 


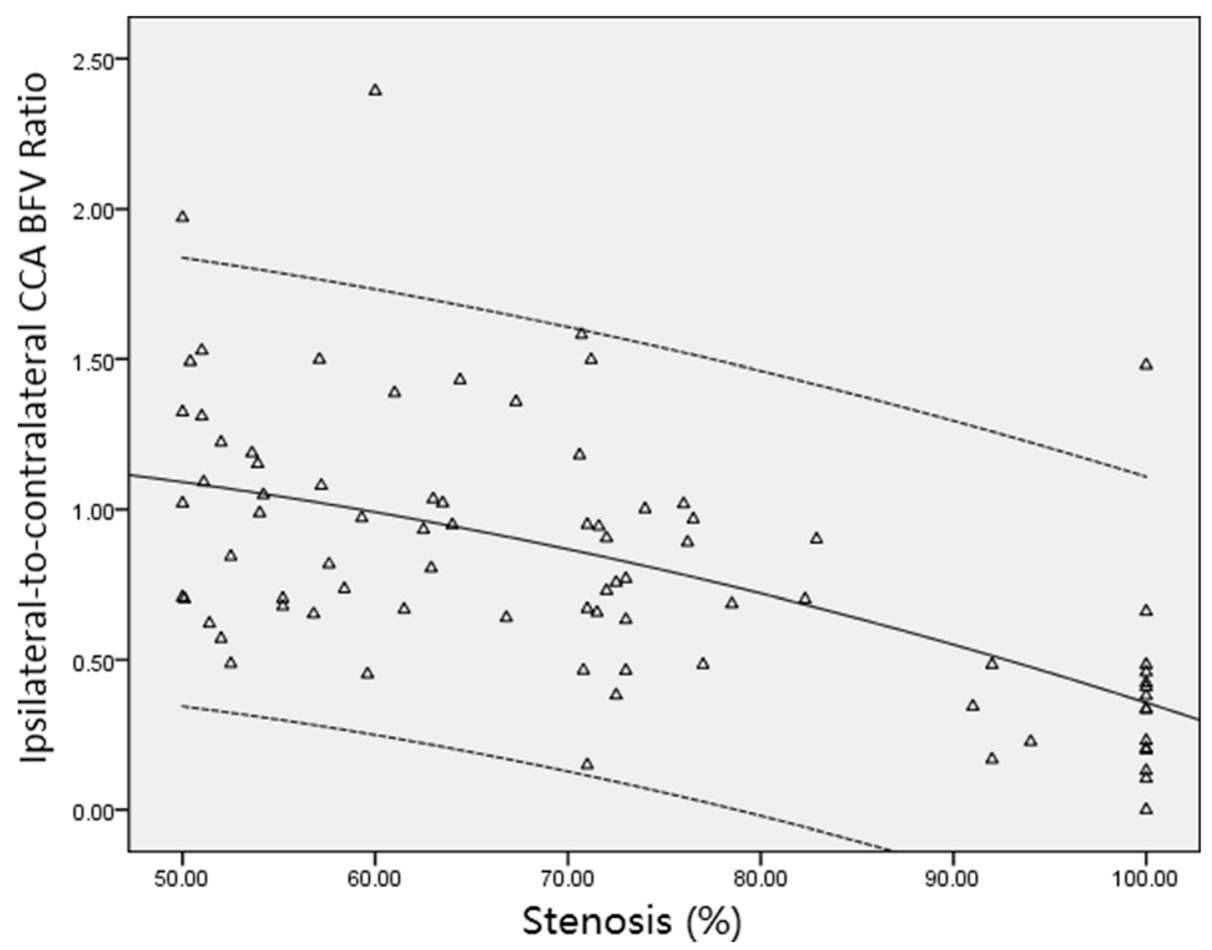

Fig. 2 A decrease in ipsilateral-to-contralateral CCA BFV Ratio is significantly associated with increasing percentage

VAs in stroke patients with unilateral carotid stenosis, indicating the presence of complete circle of Willis. While in patients with bilateral carotid stenosis, the option of supplying collateral flow through the anterior circle was insufficient, thus alternative collateral flow pathways such as the PCoAs became the most important route to contribute blood flow from the posterior circulation, which was consistent with previous MRA studies [23].

Our previous study revealed that CVIQ could accurately detect the presence of intracranial collateral circulation [8], while the present study demonstrates the cerebral blood flow redistribution from contralateral CCA and posterior circulation in patients with carotid stenosis, which reflects the compensating patterns and the presence of collateral pathways of patients with carotid occlusive disease, and illustrates that it is possible to quantify the effect of extracranial stenosis on distal cerebral circulation. What's more, the presence of adequate compensating collateral flow may protect patients from the deterioration of clinical symptoms. Therefore, flow assessment may help decision making about patient selection for interventional therapy.

Table 4 Comparisons of BFV in ipsilateral and contralateral CCA among stroke patients with unilateral ICA stenosis

\begin{tabular}{lccc}
\hline & Ipsilateral CCA & Contralateral CCA & $P$ value \\
\hline BFV & $242.2 \pm 112.2$ & $325.5 \pm 99.8$ & $<0.001$ \\
\%BFV & $34.7 \pm 12.5$ & $48.5 \pm 12.2$ & $<0.001$ \\
\hline
\end{tabular}

Compromised cerebral perfusion can be detected with various techniques, including positron emission tomography (PET), single photon emission computed tomography (SPECT), dynamic perfusion computed tomography (PCT), MRI dynamic susceptibility contrast (DSC), arterial spin labeling (ASL), and Transcranial Doppler [24]. Instead of using more popular technique to measure BFV to reflect cerebral perfusion, the present study opted to use CVIQ technique that has been validated in our laboratory, as CVIQ is more accurate in measuring cerebral blood flow volume than spectral Doppler technique and comparable to magnetic resonance angiography with phase contrast flow quantification $[9,10]$.

There are several limitations in our study. Firstly, the presence of intracranial artery stenosis was not analyzed because nearly $30 \%$ recruited patients had no proper temporal window for TCD diagnosis. Subgroup analysis will be designed to evaluate the hemodynamic effects of intracranial artery lesions on the cerebral blood flow of collateral pathway by carotid ultrasonography in patients with carotid artery stenosis. We previously found that CCA and VA contribute maximally to collateral pathways in carotid occlusive diseases [8]. Secondly, the present study did not evaluate the association of compensating patterns and risk of recurrent stroke, while further study will indicate the prognostic effect of compensating patterns on patients with carotid stenosis 
that could be used to guide recommendations for interventional therapy.

\section{Conclusion}

The present study demonstrates in stroke patients with unilateral severe carotid artery disease, contralateral carotid artery plays an important role rather than bilateral VAs in compensating reduced brain perfusion. Patients with bilateral carotid stenosis, however, fully rely on collateral flow via the posterior circulation.

\begin{abstract}
Abbreviations
ICA: internal carotid arteries; CVIQ: color velocity imaging quantification ultrasound; BFV: arterial blood flow volume; CCAs: common carotid arteries; VAs: bilateral vertebral arteries; SPECT: Single Photon Emission Computed Tomography; PWH: the Prince of Wales Hospital; TOAST: the Trial of ORG 10172 in Acute Stroke Treatment; PET: positron emission tomography; SPECT: single photon emission computed tomography; PCT: perfusion computed tomography; DSC: dynamic susceptibility contrast; ASL: arterial spin labeling.
\end{abstract}

\section{Competing interests}

The authors declare that they have no competing interests.

\section{Authors' contributions}

$H F, X Y C$ and SSYH selected the subjects and conducted the clinical evaluations. HF, BS and BC analyzed the data, performed the statistical analysis and performed the data interpretation. HF wrote the manuscript. XYC, SSYH, YMX and KSW conceived the idea of the study, interpreted the data and reviewed the manuscript. All of the authors read and approved the final manuscript. All authors critically reviewed the manuscript and approved the submitted version.

\section{Acknowledgements}

The study was supported by Direct Grant from CUHK Medicine Panel for Research 2013/2014 (4054096), grant from Shenzhen Science and Technology Innovation Committee (Project No. JC20140606164105360), and grant from the National Natural Science Foundation of China (Project No. 81371297). The funders had no role in study design, data collection and analysis, decision to publish, or preparation of the manuscript.

\section{Author details}

${ }^{1}$ Department of Neurology, the First Affiliated Hospital of Zhengzhou University, No.1 Jianshe East Road, Erqi District, Zhengzhou, Henan, PR China. ${ }^{2}$ Department of Medicine and Therapeutics, Faculty of Medicine, Prince of Wales Hospital, The Chinese University of Hong Kong, Shatin, New Territories, Hong Kong. ${ }^{3}$ Department of Imaging and Interventional Radiology, Faculty of Medicine, Prince of Wales Hospital, The Chinese University of Hong Kong, Shatin, New Territories, Hong Kong.

Received: 20 August 2015 Accepted: 10 March 2016

Published online: 18 March 2016

\section{References}

1. Rothwell PM, Eliasziw M, Gutnikov SA, Fox AJ, Taylor DW, Mayberg MR, Warlow CP, Barnett HJM. Analysis of pooled data from the randomised controlled trials of endarterectomy for symptomatic carotid stenosis. Lancet. 2003;361(9352):107-16.

2. Donahue MJ, Strother MK, Hendrikse J. Novel MRI approaches for assessing cerebral hemodynamics in ischemic cerebrovascular disease. Stroke. 2012;43(3):903-15

3. Liebeskind DS, Cotsonis GA, Saver JL, Lynn MJ, Turan TN, Cloft HJ, Chimowitz MI. Collaterals dramatically alter stroke risk in intracranial atherosclerosis. Ann Neurol. 2011;69(6):963-74

4. Gupta A, Chazen JL, Hartman M, Delgado D, Anumula N, Shao H, Mazumdar M, Segal AZ, Kamel H, Leifer D, et al. Cerebrovascular reserve and stroke risk in patients with carotid stenosis or occlusion: a systematic review and meta-analysis. Stroke. 2012;43(11):2884-91.
5. Kamouchi M, Kishikawa K, Okada Y, Inoue T, Ibayashi S, lida M. Poststenotic flow and intracranial hemodynamics in patients with carotid stenosis: transoral carotid ultrasonography study. AJNR Am J Neuroradiol. 2005;26(1):76-81.

6. Powers WJ. Cerebral hemodynamics in ischemic cerebrovascular disease. Ann Neurol. 1991;29(3):231-40

7. Maclntosh BJ, Sideso E, Donahue MJ, Chappell MA, Gunther M, Handa A, Kennedy J, Jezzard P. Intracranial hemodynamics is altered by carotid artery disease and after endarterectomy: a dynamic magnetic resonance angiography study. Stroke. 2011;42(4):979-84.

8. Ho SS, Metreweli $\mathrm{C}, \mathrm{Yu} \mathrm{CH}$. Color velocity imaging quantification in the detection of intracranial collateral flow. Stroke. 2002;33(7):1795-8.

9. Ho SS, Chan YL, Yeung DK, Metreweli C. Blood flow volume quantification of cerebral ischemia: comparison of three noninvasive imaging techniques of carotid and vertebral arteries. AJR Am J Roentgenol. 2002;178(3):551-6.

10. Ho SS, Metreweli C. Preferred technique for blood flow volume measurement in cerebrovascular disease. Stroke. 2000;31(6):1342-5.

11. Ho SS, Lam WW, Wong KS, Leung CS, Metreweli C. Potential value of post stroke extracranial arterial blood flow volume in the prediction of stroke functional outcome. Cerebrovasc Dis. 2006;21(1-2):54-9.

12. Han JH, Ho SS, Lam WW, Wong KS. Total cerebral blood flow estimated by color velocity imaging quantification ultrasound: a predictor for recurrent stroke? J Cereb Blood Flow Metab. 2007:27(4):850-6.

13. Klijn CJ, Kappelle LJ, van Schooneveld MJ, Hoppenreijs VP, Algra A, Tulleken CA, van Gijn J. Venous stasis retinopathy in symptomatic carotid artery occlusion: prevalence, cause, and outcome. Stroke. 2002;33(3):695-701.

14. Klijn CJM, Kappelle LJ. Haemodynamic stroke: clinical features, prognosis, and management. Lancet Neurol. 2010;9(10):1008-17.

15. Ogoh S, Lericollais R, Hirasawa A, Sakai S, Normand H, Bailey DM. Regional redistribution of blood flow in the external and internal carotid arteries during acute hypotension. Am J Physiol Regul Integr Comp Physiol. 2014;306(10):R747-51

16. Wada T, Kodaira K, Fujishiro K, Okamura T. Correlation of common carotid flow volume measured by ultrasonic quantitative flowmeter with pathological findings. Stroke. 1991;22(3):319-23.

17. Oktar SO, Yucel C, Karaosmanoglu D, Akkan K, Ozdemir H, Tokgoz N, Tali T. Blood-flow volume quantification in internal carotid and vertebral arteries: comparison of 3 different ultrasound techniques with phase-contrast MR imaging. AJNR Am J Neuroradiol. 2006;27(2):363-9.

18. Seidel E, Eicke BM, Tettenborn B, Krummenauer F. Reference values for vertebral artery flow volume by duplex sonography in young and elderly adults. Stroke. 1999;30(12):2692-6.

19. Grubb Jr RL, Derdeyn CP, Fritsch SM, Carpenter DA, Yundt KD, Videen TO, Spitznagel EL, Powers WJ. Importance of hemodynamic factors in the prognosis of symptomatic carotid occlusion. JAMA. 1998;280(12):1055-60.

20. Barnett HJ, Taylor DW, Eliasziw M, Fox AJ, Ferguson GG, Haynes RB, Rankin RN, Clagett GP, Hachinski VC, Sackett DL, et al. Benefit of carotid endarterectomy in patients with symptomatic moderate or severe stenosis. North American Symptomatic Carotid Endarterectomy Trial Collaborators. N Engl J Med. 1998;339(20):1415-25.

21. Randomised trial of endarterectomy for recently symptomatic carotid stenosis: final results of the MRC European Carotid Surgery Trial (ECST). The Lancet 1998, 351(9113):1379-87.

22. Caplan LR, Wong KS, Gao S, Hennerici MG. Is hypoperfusion an important cause of strokes? If so, how? Cerebrovasc Dis. 2006;21(3):145-53.

23. Hartkamp MJ, van Der Grond J, van Everdingen KJ, Hillen B, Mali WP. Circle of Willis collateral flow investigated by magnetic resonance angiography. Stroke. 1999;30(12):2671-8.

24. Wintermark M, Sesay M, Barbier E, Borbely K, Dillon WP, Eastwood JD, Glenn TC, Grandin CB, Pedraza S, Soustiel JF, et al. Comparative overview of brain perfusion imaging techniques. Stroke. 2005;36(9):e83-99. 